\title{
Video Article \\ Spontaneous and Evoked Measures of Pain in Murine Models of Monoarticular Knee Pain
}

\author{
Hollis E. Krug ${ }^{1,2}$, Christopher Dorman ${ }^{2}$, Nicole Blanshan ${ }^{2}$, Sandra Frizelle ${ }^{2}$, Maren Mahowald ${ }^{1,2}$ \\ ${ }^{1}$ Department of Medicine, University of Minnesota \\ ${ }^{2}$ Research Department, VA Health Care Center
}

Correspondence to: Hollis E. Krug at Hollis.Krug@va.gov

URL: https://www.jove.com/video/59024

DOI: doi: $10.3791 / 59024$

Keywords: Medicine, Issue 144, pain, mouse, arthritis, weightbearing, evoked, spontaneous, joint

Date Published: 2/22/2019

Citation: Krug, H.E., Dorman, C., Blanshan, N., Frizelle, S., Mahowald, M. Spontaneous and Evoked Measures of Pain in Murine Models of Monoarticular Knee Pain. J. Vis. Exp. (144), e59024, doi:10.3791/59024 (2019).

\section{Abstract}

Pain is the main cause of disability from arthritis. There is currently an unmet need for adequate treatments for arthritis pain. Pre-clinical models are necessary and useful for studying the mechanisms of pain and for evaluating efficacy of arthritis therapies. Measuring pain in animal models of arthritis is challenging. We have developed methods for measuring evoked and spontaneous pain in three models of murine arthritis. We quantitate the evoked pain responses of mice subjected to firm palpation of a painful knee. We also evaluate spontaneous pain by the proportion of weight and the amount of time placed on each of their 4 limbs after induction of arthritis pain in one knee. Joint pain in these mouse models produces a significant increase in evoked pain responses and an alteration in weight bearing. Since mice are quadrupeds, they offload the painful limb to the contralateral limb, to the forelimbs, or some combination. These methods are simple, require minimal equipment, and are reproducible and sensitive for detecting pain. They are useful for studying both disease-modifying arthritis treatments and analgesics in mice.

\section{Video Link}

The video component of this article can be found at https://www.jove.com/video/59024/

\section{Introduction}

Pain is the main cause of disability from arthritis ${ }^{1,2}$. Currently available analgesics are often ineffective, toxic, and potentially contribute to the nation-wide narcotic problem ${ }^{3}$. Surgery is not always an option for end-stage arthritis, particularly in the elderly, who may have multiple comorbidities. Intra-articular steroids and viscosupplementation may be effective in early disease but typically lose their effectiveness in late stage disease $^{4}$. There is a great unmet need for effective analgesics for arthritis pain that cannot be addressed with currently available treatments.

Pre-clinical models are useful for understanding the mechanism of action of pain therapies and for predicting limiting toxicities, but pain measurement in animals is challenging ${ }^{5}$. Pain measures can be direct and spontaneous (static or dynamic weight bearing, foot posture, gait analysis, spontaneous movement and mechanical or thermal sensitivity) or indirect/evoked (hind limb withdrawal test, knee compression force, or brain imaging) ${ }^{6}$. For mild forms of monoarthritis, gait analysis is insensitive and lacks consistency ${ }^{7}$. Static weight bearing is useful for measuring pain in rat hindlimbs but can be difficult in mice. Mechanical sensitivity measures such as the von Frey test indirectly detect sensitization of the foot pad in the setting of knee arthritis, but do not directly measure knee pain and are cumbersome to perform. Knee compression force as measured with the P.A.M. (Pressure Application Measurement) device can be used in rats, but the large device is difficult to use in mice without producing negative behaviors that do not necessarily reflect pain 8 .

We have developed methods for measuring spontaneous and evoked pain in three different models of murine arthritis that are sensitive and reproducible for measuring pain severity and the analgesic effects of intra-articular therapies ${ }^{9,10}$. We can also correlate pain relief with other histologic markers of pain and screen investigational drugs that would not be possible in humans. We can evaluate synergism of modestly effective analgesics to identify more effective combination therapies. By utilizing proven methods for both evoked and spontaneous pain, we avoid the pitfall of failing to identify pain that is reflected in one type of pain behavior but not the other and more closely mirror pain measurement in humans ${ }^{11}$. By limiting pain evaluation to two methodologies, we minimize stress on the animals, which can affect pain behavior, and maximize productivity of the laboratory.

\section{Protocol}

All animal procedures and protocols were approved by the Minneapolis Veterans Affairs Health Care System (VAHCS) Institutional Animal Care and Use Committee and conformed to the "Guide for the Care and use of Laboratory Animals" (The National Academic Press, USA) ${ }^{12}$. 


\title{
1. Choice of animals
}

\author{
NOTE: C57BI6 mice are used for all three arthritis models.
}

1. Use either male or female mice, but note that the response to arthritis pain may vary depending on gender and the pathogenesis of the model being tested.

2. Select mice that have reached sexual maturity, are no longer rapidly growing and are not old enough to have developed spontaneous arthritis that could confound pain measurements.

NOTE: Twelve weeks is an ideal age for pain measurement in the models described but this depends on the study design. Age at initiation of arthritis depends on the duration of time the model takes to develop. Acute inflammatory arthritis (carrageenan model) requires $3 \mathrm{~h}$ to develop, therefore animals are 12 weeks of age at the time of induction. Chronic inflammatory arthritis (CFA model) requires 3 weeks to develop, therefore animals are 9 weeks old at the time of induction. Chronic degenerative arthritis (collagenase model) requires 4 weeks to develop, therefore animals are 8 weeks at the time of induction. A more chronic model of degenerative arthritis can be used by inducing arthritis at 6 weeks of age. This allows 6 weeks of development of joint damage. However, chronic degenerative arthritis in mice does not produce significant evoked pain.

3. Obtain animals from a national commercial breeder source.

4. House animals in groups of up to 8 animals in standard polycarbonate cages with ad libitum rodent diet and water.

5. Maintain environmental conditions at $72{ }^{\circ} \mathrm{C} \pm 1{ }^{\circ} \mathrm{C}$ and $33 \% \pm 1 \%$ humidity with $12 \mathrm{~h}$ light/dark cycles.

\section{Intra-articular injections into the knee}

1. Create a $30 \mathrm{G} 2.5 \mathrm{~mm}$ sterile by covering the length of a 0.5 inch needle with a sheath made of polyethylene tubing with an inside diameter of $0.38 \mathrm{~mm}$ and outside diameter of $1.09 \mathrm{~mm}$, leaving only $2.5 \mathrm{~mm}$ of the needle protruding. This prevents inserting the needle too deeply and exiting the joint posteriorly.

2. Choose a single knee to inject, shave it using any commercially available electric facial hair trimmer, and sterilize with providone-iodine scrub.

3. Anesthetize the mouse with $3 \%$ isoflurane in $100 \% \mathrm{O} 2$ at $1 \mathrm{~L} / \mathrm{min}$ for $3 \mathrm{~min} \pm 1 \mathrm{~min}$. Maintain anesthesia using a nosecone during the procedure. Confirm anesthesia depth by toe pinch. Take care to monitor respiratory status and minimize anesthesia exposure to maintain adequate respirations.

4. Enter the joint at the midline through the patellar tendon just inferior to the lower edge of the patella using the sterile sheathed needle once the mouse is fully anesthetized. This anterior midline approach maximizes accuracy for entering the joint and injecting the substance of interest.

5. Use the same approach for injecting both arthritis-inducing substances and analgesic substances. Sham controls are produced by inserting the needle as above but without injecting any substance.

\section{Production of arthritis}

1. Anesthetize animal as in step 2.3 above.

2. Weigh the appropriate amounts (see below) of carrageenan, Complete Freund's Adjuvant and Type IV Collagenase and dilute to the appropriate concentration with normal saline in a laminar flow hood for safety and sterility.

3. Inject $10 \mu \mathrm{l}$ of $2.5 \%$ carrageenan diluted in $0.9 \%$ sterile saline into the knee as above using a Hamilton syringe to produce acute inflammatory arthritis. Arthritis peaks $3 \mathrm{~h}$ after injection.

4. Inject, $30 \mu \mathrm{L}(30 \mu \mathrm{g})$ of Complete Freund's Adjuvant (CFA) into the knee to produce chronic inflammatory arthritis. There is an initial acute inflammatory phase that peaks at 1 week then a chronic phase that peaks at 3 weeks following injection.

5. Inject $10 \mu \mathrm{L}$ containing 10 IU Type IV Collagenase to produce chronic degenerative arthritis. Arthritis pain peaks 4 weeks after injection.

\section{Measurement of evoked joint pain}

1. Always examine the normal comparator knee first to minimize the cross-over pain response (windup) from the painful knee ${ }^{13}$.

2. Train the examiner to apply consistent and firm pressure of $700 \mathrm{gf} / \mathrm{cm}^{2}=10 \mathrm{psi}$ using a Palpometer level 4 ; the device will emit 4 beeps when this level of pressure is applied. This amount of pressure will consistently elicit a greater pain response from an arthritic joint than from a normal joint.

NOTE: The device is calibrated at the factory to elicit 4 beeps when the appropriate level of pressure is applied. Use the device for training only; do not use on the animals but when examining the animals, apply the amount of pressure learned through the training.

3. Restrain the mouse tightly enough to prevent casual movement by holding the scruff of the neck with the index finger and thumb and using the $4^{\text {th }}$ and $5^{\text {th }}$ fingers to restrain the tail. The mouse should be able to make discrete, brief movements with effort and when stimulated, but should not be able to move freely or continuously.

4. Administer repeated firm (Palpometer level 4) palpations concurrently to the lateral and medial sides of each knee at approximately the joint line with the thumb and index finger of the free hand once per second for one minute while restraining the mouse. Do not use the Palpometer for the mouse examination; it is used only for training.

5. Have a second observer count the number of times the animal vocalizes and fights to escape the restraint during this one-minute time-period. The sum of fights and vocalizations is the Evoked Pain Score.

6. Always us the same examiner throughout the study and confirm the examiner and observer are blinded as to experimental condition. 


\section{Measurement of spontaneous joint pain.}

NOTE: The Advanced Dynamic Weight Bearing (ADWB) device calculates the percent of total time the animal spends on each limb and the percent of total weight borne on each limb.

1. Fill a plastic beaker with $2 \mathrm{~L}$ of water. Place the foam pad and medium density fiberboard that are included with the equipment along with the filled beaker on a laboratory scale and record the combined weight.

2. Open the ADWB program and select Mice sensor type.

3. Calibrate the system prior to each experiment:

1. Select Full calibration under the Calibration menus. Enter 0.00 as a Tare value and the weight of the foam pad, medium density fiberboard, plastic beaker, and water as the Calibration weight, then select Next.

2. Select Tare before placing anything on the sensor. Before selecting Gain preview, cover the sensor pad with the provided foam pad, medium density fiberboard, and plastic beaker filled with 2 liters of water, in that order. Select Gain preview once the calibration weight is on the sensor.

3. Adjust the calibration weight so it is evenly distributed across the individual sensors and when the value of the Calibration indicator is above 95, select Gain definition. This process produces a normalized mean for the individual sensors and confirms the integrity of the individual sensors. Finally, select Next.

4. Save the calibration file into the calibration folder.

4. Select New and where provided enter the Project name and the Animal name for the first mouse to be tested.

5. Select Browse and open the calibration file that was created for the project. Select Next, ensure Enable video capture is selected, and select OK.

6. Weigh the animal, select Capture, and record the weight in the software. Select OK.

7. Do not acclimate mice to the chamber before the collection of data because this device measures spontaneous behavior

8. Place mouse in the chamber for a period of $5 \mathrm{~min}$ of testing. When the animal is in the chamber select Launch. The software will record the animal's activity during this time, capture a video and synchronize with the sensor data, and automatically partition this video into analyzable and non-analyzable segments.

NOTE: The software considers segments analyzable when at least one stable zone is detected. A stable zone is created when sensors detect $\geq 2 \mathrm{~g}$ on one sensor with a minimum of one adjacent sensor detecting $\geq 1 \mathrm{~g}$ and when the weight distribution of a zone is stable for more than $0.38 \mathrm{~s}$

9. Before the first analysis in a series select Easy scoring and Independent paws setting

NOTE: Easy scoring allows iteration validation of segments where the stable zone does not change based on the validation of the first stable zone of that segment. Independent paws setting provides the observer with access to the keyboard shortcuts that allow individual identification of each paw. The observer assigns zones that meet minimal criteria as right hind, left hind, right fore, left fore, both forepaws, other body part, or ignore zone by using the keyboard shortcuts while holding the cursor over the segment per the video and scaled map of activated sensors.

10. Select $\gg$ to move to the first analyzable segment. If a stable zone does not have the correct paw identified then hold the cursor over that zone and press the shortcut (right hind, $\mathbf{s}$; left hind, $\mathbf{a}$; right fore, $\mathbf{w}$; left fore, $\mathbf{q}$; both forepaws, $\mathbf{e}$; other section of the body, $\mathbf{z}$, ignore zone, $\mathbf{x}$ ) that corresponds with the correct body part.

NOTE: A minimum of $1.5 \mathrm{~min}$ of analyzable time is required to meet analysis thresholds. Analyzed segments are added and averaged automatically for output per paw(s) by the software.

11. The ADWB software then automatically calculates the percentage of analyzed time spent and percentage of body weight (g) placed on each of the four limb options. Export these values into a new spreadsheet by selecting Results, Export, selecting Yes to the prompt question asking if only the first five summary lines should be exported, and selecting the location of the output.

12. Euthanize animals by $\mathrm{CO} 2$ inhalation followed by thoracotomy at the end of the study period.

13. For each additional animal in the same group, select New and enter a unique animal name but do not change the project name. Select Next, ensure Enable video capture is selected, and select OK. Follow steps 5.5 to 5.12 as for the first animal.

\section{Representative Results}

Acute and chronic inflammatory and chronic degenerative joint pain was produced by IA injection into the left knee of C57BL/6J male and female mice prior to pain assessment using the protocol outlined above. Joint pain was measured as evoked pain scores (EPS) and spontaneous pain behaviors measured with an advanced dynamic weight bearing (ADWB) device. For the purposes of this report, individual comparisons were made using Student's t tests. For experiments where analgesic doses are being compared, ANOVA may be a more appropriate statistical comparison.

EPS (the sum of tallied fights plus vocalizations) increased with all three types of joint pain (Figure 1). ADWB measures of percent weight bearing on the left hind limb compared to naïve mice were reduced in acute and chronic inflammatory joint pain. Females but not males with chronic degenerative joint pain (COL) showed similar reductions in ADWB measures (Figure 2A). Males with acute inflammatory joint pain transferred more weight to the forelimbs than naïve animals. Weight bearing on forelimbs increased in female mice compared to naïve but not in males with chronic degenerative joint pain (COL) (Figure 2B). ADWB measures of percent time on the affected left hind limb decreased compared to naïve in mice with acute and chronic inflammatory joint pain, but this was not statistically significant. These animals did spend significantly less time on the left hind limb than on the right hind limb. In both males and females with chronic degenerative joint pain, there was no difference in time spent on the left hind limbs compared to naïve. Females with chronic degenerative joint pain spent more time on the nonarthritic right hind limb compared to naive (Figure 3A). Mice with acute inflammatory joint pain and females with chronic degenerative joint pain spent significantly more time on the forelimbs than naïve animals (Figure 3B). 


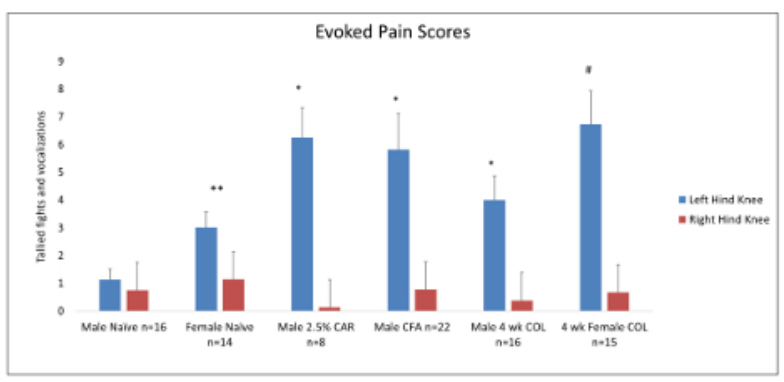

Figure 1. Evoked pain scores for left (blue column) and right (red column) hind limbs. Right hind limbs served as internal controls and did not undergo any IA injections. Measurements were taken in the following conditions: uninjected naïve (male and female), acute inflammatory carrageenan-induced arthritis (CAR), chronic inflammatory Complete Freund's Adjuvant-induced arthritis (CFA) and chronic noninflammatory Collagenase-induced arthritis (COL - male and female). In naïve animals the EPS of the left hind knee was not statistically different from the right in males and only minimally different in females $\left({ }^{* *} P=0.038\right.$ ) likely due to some increased pain response in the knee examined second (the left) due to crossover pain response. In all arthritic groups the arthritic left knee EPS was significantly greater than the nonarthritic contralateral right knee. When comparing the EPS of the left knee of arthritis groups to naïve animals, all arthritis groups had significantly greater EPS scores in the arthritic left knee compared to naïve left knees. This was true in both males and females. ${ }^{*} P<0.05$ compared to male naïve, \#P<0.05 compared to female naïve. All error bars are SEM in all figures. Please click here to view a larger version of this figure.

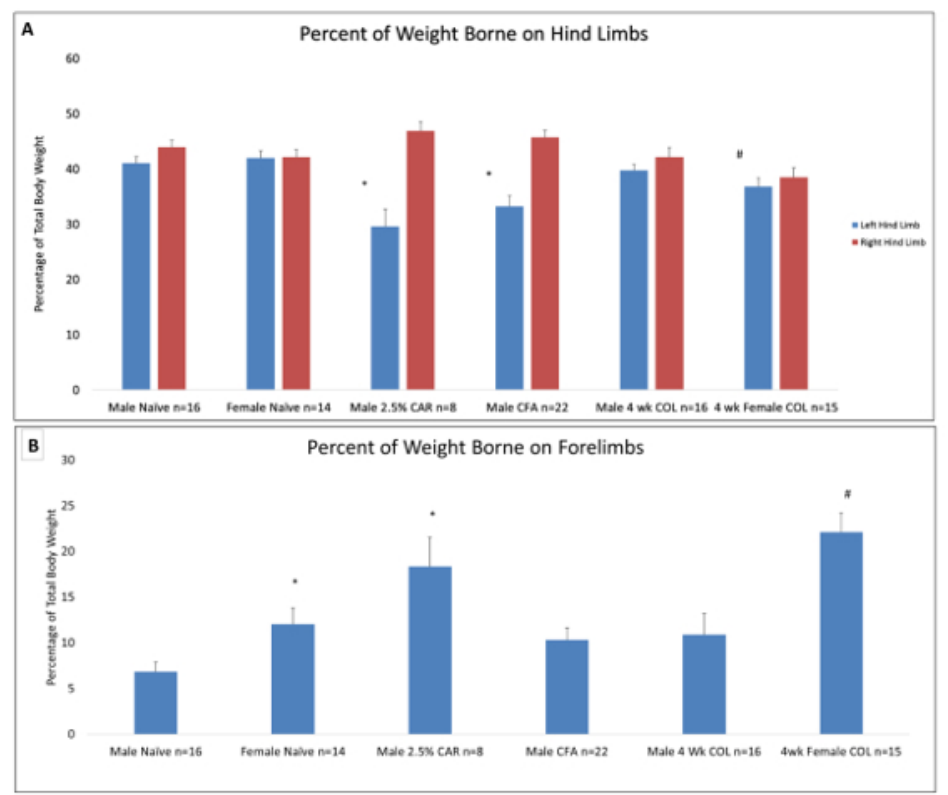

Figure 2: Spontaneous pain behavior measured by percent body weight borne on limbs. (A) Spontaneous pain behavior measured by total percent body weight on the left (blue column) and right (red column) hind limbs. In both male and female naïve animals, there was no significant difference between percent weight borne on the left and right hind limbs. Male mice with CAR and CFA induced joint pain and females with COL induced joint pain bore significantly less weight on the left hind limb compared to the naive. ${ }^{*} P<0.05$ compared to male naïve, $\# P<0.05$ compared to female naïve. (B) Spontaneous pain behavior measured by total percent body weight placed on the forelimbs. In males with CAR induced acute joint pain and females with $\mathrm{COL}$ induced chronic joint pain, there was a significant increase in the amount of weight borne on the forelimbs. This was not seen in males with CFA or COL induced joint pain. Naïve females tended to bear more weight on the forelimbs than naïve males $(P=0.02)$. Additional weight may be borne by other body parts such as the tail or rump. These were usually minimal and did not contribute significantly to the understanding of the pain response and are therefore not shown. ${ }^{*} P<0.05$ compared to male naïve, $\# P<0.05$ compared to female naïve and to male 4 week $\mathrm{COL}$ in forelimb weightbearing. Please click here to view a larger version of this figure. 


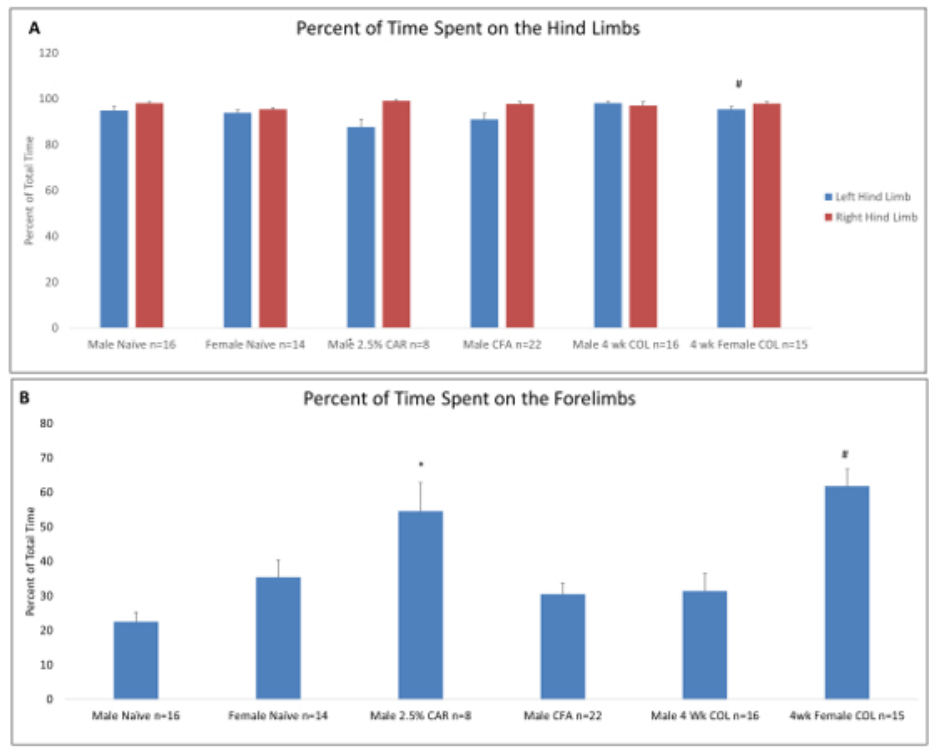

Figure 3: Spontaneous pain behavior measured by percent time spent on limbs. (A) Spontaneous pain behavior measured by total percent time spent on the left (blue column) and right (red column) hind limbs. There was no significant difference between right and left hind limbs in male and female naïve mice with respect to time spent on the limb. Male mice with CAR and CFA induced arthritis appeared to spend less time weightbearing with the arthritic left hind limb compared to naïve, but this was not statistically significant and likely due to increased variability in this measure in these mice. There was a significant difference in these animals comparing right to left as the right hind limb spent more total time weightbearing than the left. In females with $\mathrm{COL}$ induced arthritis, the right hind limb was weightbearing a greater proportion of time than in naïve females. ${ }^{*} P<0.05$ comparing right to left, $\# P<0.05$ compared to female naïve and to male 4 week COL in time spent on forelimbs. (B) Spontaneous pain behavior measured by total amount of time spent on the forelimbs. In males with CAR induced arthritis and females with COL induced arthritis the mice spent significantly more time weightbearing on the fore limbs than did the naïve animals. Female naïve mice spent more time on the forelimbs than male naïve animals. ${ }^{\star} P<0.05$ compared to male naïve, $\# P<0.05$ compared to female naïve. Please click here to view a larger version of this figure.

\section{Discussion}

Measurement of joint pain in preclinical models of inflammatory arthritis and degenerative joint disease is an increasingly valuable tool to evaluate new treatments for arthritis pain and for restoring joint function ${ }^{9,10}$. Measuring pain behaviors in mice with chronic degenerative joint disease is challenging. As quadrupeds, they can offload a painful limb to any combination of the other three limbs or the tail. Decreased function in one limb may not be displayed as obviously as it is in bipedal humans. As prey animals, mice have a survival benefit to minimize pain behaviors.

This report demonstrates that joint pain from different pathologic mechanisms can be measured in mice with simple and relatively inexpensive methods. Inflammatory joint pain causes bigger changes in weight bearing time and distribution and therefore may be a more sensitive model for the detection of analgesia. However, other models of joint pain will be important when evaluating new potential analgesics since mechanisms of pain initiation and maintenance are likely different with different pathology. We found differences in offloading patterns between males and females and among different models of arthritis pain.

It is important to recognize that there can be strain as well as gender differences in pain behavior in mice ${ }^{14,15,16}$. It is also known that there are distinct strain differences in pain thresholds. We found that in C57/BI6 mice, females demonstrated significantly more spontaneous pain behaviors (increased weight and time on forelimbs) than male mice with chronic degenerative joint pain (Figure 2 and Figure 3). We did not determine the estrus phase for these female mice, so we cannot say whether this had any effect on our results, but others have determined that estrus phase did not explain observed sex differences in C57/BI6 mice ${ }^{17}$.

Evoked pain behavior is sensitive to all types of joint pain tested and has good reproducibility. It is crucial that the examiner is trained to deliver consistent, standardized palpation pressure and use consistent restraint methods that allows the range of pain behavior to detect both pain and analgesia. It is important to always examine the normal knee first to minimize the pain response in the normal knee due to a crossover effect and maximize the difference in pain response between the normal and painful knee. Using a second observer to tally the pain responses that make up the EPS is necessary so that the examiner focuses on consistent palpation and so that vocalizations and fights are consistently tallied. It may be necessary to practice the restraint method in particular to ensure the animal can react to pain adequately but does not react too easily so as to dilute the pain response. An advantage of the EPS measurement is that it is analogous to measuring joint tenderness in human patients.

We feel these methods are superior to existing methods in that they directly measure pain coming from the knee joints of mice, they can be used in different models of monoarthritis pain, they include both spontaneous and evoked pain responses, and are relatively inexpensive but reliable and reproducible. As therapies are developed to more effectively treat arthritis, these methods will be useful for confirming whether treatments are actually effective for the most disabling outcome of arthritis, pain. 
Our methods have some limitations. Primarily, the EPS measure is limited by the requirement for a single examiner and observer and the need for very consistent technique. The evoked pain measures are more automated and are not as subject to inter-observer differences. Further refinement of the EPS measure might include automated methods for counting vocalizations in response to palpation, such as using an ultrasonic bat detector to count vocalizations. This method would need to be compared to our current method to confirm sensitivity and specificity before it could be widely implemented.

In summary, we describe two different pain behavior measures for quantifying joint pain in mice. Gender and strain differences must be considered before choosing an experimental model. Measures of evoked pain require careful training and consistent implementation by the examiner. Both spontaneous and evoked pain behaviors are recommended to evaluate pain severity and functional limitation and should be utilized to fully evaluate the effects of new potential analgesics.

\section{Disclosures}

The authors have nothing to disclose.

\section{Acknowledgments}

Funded by the Department of Veterans Affairs MERIT award 2 I01 RX000379-05.

\section{References}

1. Neggi, T. The epidemiology and impact of pain in osteoarthritis. Osteoarthritis and Cartilage. 21, 1145-1153 (2013).

2. Center for Disease Control and Prevention. Prevalence of doctor-diagnosed arthritis and arthritis-attributable activity limitation - United States, 2007-2009. Morbidity and Mortality Weekly Report. 59, 1261-1265 (2010).

3. Kaye, A.et al. Prescription Opioid Abuse in Chronic Pain: An Updated Review of Opioid Abuse Predictors and Strategies to Curb Opioid Abuse: Part 1. Pain Physician. 20, S93-S109 (2017).

4. Maheu, E.et al. Why we should definitely include intra-articular hyaluronic acid as a therapeutic option in the management of knee osteoarthritis: Results of an extensive critical literature review. Seminars in Arthritis and Rheumatism. (2018).

5. Malfait, A.-M. Why we should study pain in animal models of rheumatic diseases. Clinical and Experimental Rheumatology. 35, S37-S39 (2017).

6. Piel, M. J., Kroin, J. S., van Wijnen, A. J., Kc, R., Im, H.-J. Pain Assessment in Animal Models of Osteoarthritis. Gene. 537, 184-188 (2014).

7. Dorman, C. W., Krug, H. E., Frizelle, S. P., Funkenbusch, S., Mahowald, M. L. A comparison of DigiGait ${ }^{\mathrm{TM}}$ and TreadScan ${ }^{\mathrm{TM}}$ imaging systems: assessment of pain using gait analysis in murine monoarthritis. Journal of Pain Research. 7, 25-35 (2013).

8. Barton, N.et al. Pressure application measurement (PAM): A novel behavioural technique for measuring hypersensitivity in a rat model of joint pain. Journal of Neuroscience Methods. 163, 67-75 (2007).

9. Bert, J.et al. The Effect of Treatment with Resiniferatoxin and Capsaicin on Dynamic Weight Bearing Measures and Evoked Pain Responses in a Chronic Inflammatory Arthritis Murine Model. Internal Medicine Review. 2016 (6), 89 (2016).

10. Abdullah, M.et al. The effect of intra-articular vanilloid receptor agonists on pain behavior measures in a murine model of acute monoarthritis. Journal of Pain Research. 9, 563-570 (2016).

11. Anke, T. T., Kuner, R. Studying ongoing and spontaneous pain in rodents - challenges and opportunities. European Journal of Neuroscience. 39, 1881-1890, (2014).

12. National Research Council. Guide for the Care and Use of Laboratory Animals. National Academies Press, (2011).

13. Kuner, R. Central mechanisms of pathological pain. Nature Medicine. 16, 1258-1266 (2010).

14. Banik, R., Woo, Y., Park, S., Brennan, T. Strain and Sex Influence on Pain Sensitivity after Plantar Incision in the Mouse. Anesthesiology. 105 1246-1252 (2006).

15. Mogil, J.S., Chesler, E.J., Wilson, S.G., Juraska, J.M., Sternberg, W.F. Sex differences in thermal nociception and morphine antinociception in rodents depend on genotype. Neuroscience \& Biobehavioral Reviews. 24, 375-389 (2000).

16. Mogil, J.et al. Heritability of nociception I: Responses of 11 inbred mouse strains on 12 measures of nociception. Pain Physician. 80, 67-82 (1999).

17. Meziane, H., Ouagazzal, A.-M., Aubert, L., Wietrzych, M., Krezel, W. Estrous cycle effects on behavior of C57BL/6J and BALB/cByJ female mice: implications for phenotyping strategies. Genes, Brain, and Behavior. 6, 192-200 (2007). 